\title{
Experimental and CPA EoS Description of the Key Components in the BTX Separation from Gasolines by Extractive Distillation with Tricyanomethanide-based Ionic Liquids
}

Miguel Ayuso $^{a}$, André M. Palma ${ }^{b}$, Marcos Larriba ${ }^{a, b}$, Noemí Delgado-Mellado ${ }^{a}$, Julián

García $^{a}$, Francisco Rodríguez $^{a}$, João A.P. Coutinho ${ }^{b}$, Pedro J. Carvalho ${ }^{b}$, Pablo Navarro $^{b, c, *}$

${ }^{a}$ Department of Chemical Engineering, Complutense University of Madrid, Madrid, Spain.

${ }^{b}$ CICECO - Aveiro Institute of Materials, Department of Chemistry, University of Aveiro, Aveiro, Portugal.

${ }^{c}$ Department of Chemical Engineering, Autónoma University of Madrid, Madrid, Spain.

\section{Supporting Information}

\footnotetext{
* To whom correspondence should be addressed. E-mail address: pablo.navarro@uam.es (Pablo Navarro).
} 
Table S1. CPA EoS Binary Interaction Parameters $\left(k_{i j}\right)$ for $\{$ Aliphatic (1) + Aromatic (2) + IL (3)\} Systems as Function of Temperature

\begin{tabular}{|c|c|c|c|c|c|c|}
\hline \multicolumn{7}{|c|}{$k_{\mathrm{i} 3}$} \\
\hline $\mathrm{T} / \mathrm{K}$ & $n$-hexane & $n$-heptane & $n$-octane & benzene & toluene & $p$-xylene \\
\hline \multicolumn{7}{|l|}{$\left[\mathrm{C}_{2} \mathrm{C}_{1} \mathrm{im}\right][\mathrm{TCM}]$} \\
\hline 323.2 & 0.010 & 0.000 & -0.040 & -0.060 & -0.045 & -0.045 \\
\hline 363.2 & 0.020 & 0.010 & -0.030 & -0.055 & -0.045 & -0.040 \\
\hline 403.2 & 0.030 & 0.020 & -0.020 & -0.050 & -0.035 & -0.035 \\
\hline Correction 403.2 (ternary) & -0.050 & -0.060 & - & -0.090 & -0.055 & - \\
\hline \multicolumn{7}{|l|}{$\left[4-\mathrm{C}_{4} \mathrm{C}_{1} \mathrm{py}\right][\mathrm{TCM}]$} \\
\hline 323.2 & 0.045 & 0.040 & 0.015 & -0.050 & -0.040 & -0.025 \\
\hline 363.2 & 0.050 & 0.045 & 0.020 & -0.040 & -0.035 & -0.020 \\
\hline 403.2 & 0.055 & 0.055 & 0.025 & -0.035 & -0.020 & -0.015 \\
\hline Correction 403.2 (ternary) & 0.000 & 0.000 & - & -0.060 & -0.050 & - \\
\hline \multicolumn{7}{|c|}{$k_{12}$} \\
\hline Binary systems & $n$-hexane $(1)+$ benzene $(2)$ & $n$-heptane (1) + benzene (2) & $n$-octane (1) + benzene (2) & $n$-heptane $(1)+$ toluene $(2)$ & $n$-octane (1) + toluene (2) & $n$-octane $(1)+p$-xylene (2) \\
\hline 323.2 & 0.013 & 0.007 & 0.010 & 0.013 & 0.007 & 0.013 \\
\hline 363.2 & 0.010 & 0.007 & 0.010 & 0.010 & 0.007 & 0.010 \\
\hline 403.2 & 0.007 & 0.007 & 0.010 & 0.007 & 0.007 & 0.007 \\
\hline
\end{tabular}


Table S2. Vapor-liquid and Vapor-liquid-liquid Equilibria ${ }^{a}$ for $n$-Octane (1) + Benzene $(2)+\left[C_{2} C_{1}\right.$ im] $[$ TCM] (3)

\begin{tabular}{|c|c|c|c|c|c|c|c|c|c|c|c|}
\hline$p / \mathbf{k P a}$ & $y_{1}$ & $y_{2}$ & $x_{1, \mathrm{I}}$ & $x_{2, \mathrm{I}}$ & $x_{1, \mathrm{II}}$ & $x_{2, \mathrm{II}}$ & $x_{3, \mathrm{II}}$ & $x_{1}$ & $x_{2}$ & $x_{3}$ & $\alpha_{12}$ \\
\hline \multicolumn{12}{|c|}{$T / \mathrm{K}=323.2$} \\
\hline 8.9 & 0.0000 & 1.0000 & & & & & & 0.0000 & 0.1963 & 0.8037 & \\
\hline 13.4 & 0.3643 & 0.5494 & & & & & & 0.0115 & 0.1843 & 0.8042 & 10.6 \\
\hline 13.1 & 0.3938 & 0.5494 & 0.8009 & 0.1991 & 0.0075 & 0.1645 & 0.8280 & 0.0267 & 0.1655 & 0.8079 & 4.4 \\
\hline 12.3 & 0.4506 & 0.5494 & 0.8323 & 0.1677 & 0.0074 & 0.1419 & 0.8508 & 0.0410 & 0.1431 & 0.8160 & 2.9 \\
\hline 11.1 & 0.4987 & 0.5013 & 0.8558 & 0.1442 & 0.0074 & 0.1221 & 0.8706 & 0.0561 & 0.1235 & 0.8204 & 2.2 \\
\hline 10.7 & 0.5663 & 0.4337 & 0.8745 & 0.1255 & 0.0073 & 0.1045 & 0.8882 & 0.0736 & 0.1062 & 0.8202 & 1.9 \\
\hline 9.7 & 0.6155 & 0.3845 & 0.9045 & 0.0955 & 0.0076 & 0.0803 & 0.9122 & 0.0849 & 0.0817 & 0.8334 & 1.5 \\
\hline 9.0 & 0.6842 & 0.3158 & 0.9280 & 0.0720 & 0.0071 & 0.0603 & 0.9326 & 0.1010 & 0.0615 & 0.8375 & 1.3 \\
\hline 8.1 & 0.7708 & 0.2292 & 0.9553 & 0.0447 & 0.0070 & 0.0363 & 0.9568 & 0.1166 & 0.0373 & 0.8461 & 1.1 \\
\hline 7.4 & 0.8713 & 0.1287 & 0.9765 & 0.0235 & 0.0071 & 0.0194 & 0.9735 & 0.1300 & 0.0200 & 0.8501 & 1.0 \\
\hline 6.7 & 1.0000 & 0.0000 & 1.0000 & 0.0000 & 0.0071 & 0.0000 & 0.9929 & 0.1453 & 0.0000 & 0.8547 & \\
\hline \multicolumn{12}{|c|}{$T / \mathrm{K}=363.2$} \\
\hline 47.2 & 0.0000 & 1.0000 & & & & & & 0.0000 & 0.1745 & 0.8255 & \\
\hline 53.4 & 0.3042 & 0.6958 & & & & & & 0.0042 & 0.1659 & 0.8300 & 17.3 \\
\hline 58.4 & 0.4421 & 0.5579 & & & & & & 0.0130 & 0.1477 & 0.8393 & 9.0 \\
\hline 54.3 & 0.4870 & 0.5130 & & & & & & 0.0274 & 0.1300 & 0.8425 & 4.5 \\
\hline 51.1 & 0.5431 & 0.4569 & & & & & & 0.0410 & 0.1112 & 0.8479 & 3.2 \\
\hline 46.8 & 0.6005 & 0.3995 & 0.8931 & 0.1069 & 0.0067 & 0.0910 & 0.9023 & 0.0556 & 0.0928 & 0.8516 & 2.5 \\
\hline 44.4 & 0.6660 & 0.3340 & 0.9161 & 0.0839 & 0.0070 & 0.0738 & 0.9192 & 0.0697 & 0.0753 & 0.8550 & 2.2 \\
\hline 41.6 & 0.7419 & 0.2581 & 0.9342 & 0.0658 & 0.0069 & 0.0557 & 0.9374 & 0.0854 & 0.0572 & 0.8574 & 1.9 \\
\hline 38.3 & 0.8306 & 0.1694 & 0.9587 & 0.0413 & 0.0064 & 0.0340 & 0.9596 & 0.1015 & 0.0351 & 0.8633 & 1.7 \\
\hline 36.3 & 0.9042 & 0.0958 & 0.9782 & 0.0218 & 0.0066 & 0.0185 & 0.9749 & 0.1168 & 0.0191 & 0.8641 & 1.5 \\
\hline 33.6 & 1.0000 & 0.0000 & 1.0000 & 0.0000 & 0.0072 & 0.0000 & 0.9928 & 0.1323 & 0.0000 & 0.8677 & \\
\hline
\end{tabular}

a Standard uncertainty $(u)$ are $u(y)=0.001, u(x)=0.001, u\left(x_{\mathrm{I}}\right)=0.02, u\left(x_{\mathrm{II}}\right)=0.002, u_{\mathrm{r}}(P)=0.02$, and $u(T)=0.1 \mathrm{~K}$ 
Table S2. Continued

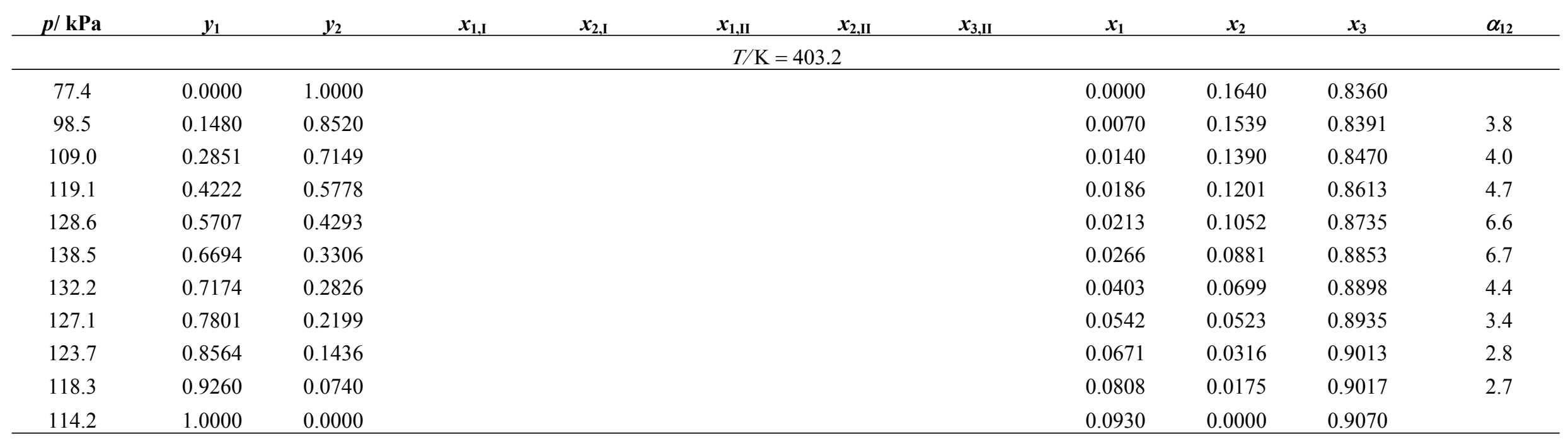

${ }^{a}$ Standard uncertainty $(u)$ are $u(y)=0.001, u(x)=0.001, u\left(x_{\mathrm{I}}\right)=0.02, u\left(x_{\mathrm{II}}\right)=0.002, u_{\mathrm{r}}(P)=0.02$, and $u(T)=0.1 \mathrm{~K}$. 
Table S3. Vapor-liquid and Vapor-liquid-liquid Equilibria ${ }^{a}$ for $n$-Octane (1) + Benzene (2) $+\left[4-C_{4} C_{1}\right.$ py][TCM] (3)

\begin{tabular}{|c|c|c|c|c|c|c|c|c|c|c|c|}
\hline$p / \mathbf{k P a}$ & $y_{1}$ & $y_{2}$ & $x_{1, \mathrm{I}}$ & $x_{2, \mathrm{I}}$ & $x_{1, \mathrm{II}}$ & $x_{2, \mathrm{II}}$ & $x_{3, \mathrm{II}}$ & $x_{1}$ & $x_{2}$ & $x_{3}$ & $\alpha_{12}$ \\
\hline \multicolumn{12}{|c|}{$T / \mathrm{K}=323.2$} \\
\hline 8.5 & 0.0000 & 1.0000 & & & & & & 0.0000 & 0.2319 & 0.7681 & \\
\hline 9.5 & 0.3666 & 0.6334 & & & & & & 0.0142 & 0.2138 & 0.7720 & 8.7 \\
\hline 10.7 & 0.5122 & 0.4878 & & & & & & 0.0301 & 0.1910 & 0.7789 & 6.7 \\
\hline 10.5 & 0.5551 & 0.4449 & 0.8472 & 0.1528 & 0.0130 & 0.1673 & 0.8197 & 0.0470 & 0.1668 & 0.7861 & 4.4 \\
\hline 10.2 & 0.5833 & 0.4167 & 0.8685 & 0.1315 & 0.0129 & 0.1449 & 0.8422 & 0.0647 & 0.1442 & 0.7911 & 3.1 \\
\hline 9.3 & 0.6400 & 0.3600 & 0.8920 & 0.1080 & 0.0131 & 0.1209 & 0.8660 & 0.0824 & 0.1200 & 0.7976 & 2.6 \\
\hline 8.7 & 0.6907 & 0.3093 & 0.9127 & 0.0873 & 0.0128 & 0.0976 & 0.8896 & 0.0996 & 0.0967 & 0.8037 & 2.2 \\
\hline 8.3 & 0.7495 & 0.2505 & 0.9345 & 0.0655 & 0.0132 & 0.0727 & 0.9141 & 0.1171 & 0.0720 & 0.8109 & 1.8 \\
\hline 7.9 & 0.8223 & 0.1777 & 0.9602 & 0.0398 & 0.0133 & 0.0448 & 0.9419 & 0.1368 & 0.0442 & 0.8190 & 1.5 \\
\hline 7.3 & 0.9045 & 0.0955 & 0.9784 & 0.0216 & 0.0129 & 0.0243 & 0.9628 & 0.1543 & 0.0239 & 0.8218 & 1.5 \\
\hline 6.7 & 1.0000 & 0.0000 & 1.0000 & 0.0000 & 0.0137 & 0.0000 & 0.9863 & 0.1725 & 0.0000 & 0.8275 & \\
\hline \multicolumn{12}{|c|}{$T / \mathrm{K}=363.2$} \\
\hline 25.8 & 0.0000 & 1.0000 & & & & & & 0.0000 & 0.2075 & 0.7925 & \\
\hline 34.9 & 0.2677 & 0.7323 & & & & & & 0.0089 & 0.1887 & 0.8024 & 7.8 \\
\hline 42.0 & 0.4548 & 0.5452 & & & & & & 0.0181 & 0.1689 & 0.8130 & 7.8 \\
\hline 48.1 & 0.5890 & 0.4110 & & & & & & 0.0194 & 0.1222 & 0.8585 & 9.0 \\
\hline 45.6 & 0.6453 & 0.3547 & 0.8801 & 0.1199 & 0.0084 & 0.1257 & 0.8659 & 0.0425 & 0.1264 & 0.8311 & 5.4 \\
\hline 43.4 & 0.6868 & 0.3132 & 0.8947 & 0.1053 & 0.0085 & 0.1080 & 0.8836 & 0.0614 & 0.1087 & 0.8299 & 3.9 \\
\hline 42.0 & 0.7138 & 0.2580 & 0.9036 & 0.0964 & 0.0090 & 0.0865 & 0.9045 & 0.0767 & 0.0870 & 0.8363 & 3.3 \\
\hline 40.7 & 0.7420 & 0.2580 & 0.9172 & 0.0828 & 0.0094 & 0.0664 & 0.9242 & 0.0955 & 0.0666 & 0.8379 & 2.8 \\
\hline 36.5 & 0.8684 & 0.1316 & 0.9598 & 0.0402 & 0.0094 & 0.0412 & 0.9494 & 0.1164 & 0.0415 & 0.8421 & 2.4 \\
\hline 35.2 & 0.9269 & 0.0731 & 0.9777 & 0.0223 & 0.0093 & 0.0219 & 0.9688 & 0.1314 & 0.0222 & 0.8464 & 2.1 \\
\hline 33.4 & 1.0000 & 0.0000 & 1.0000 & 0.0000 & 0.0095 & 0.0000 & 0.9905 & 0.1468 & 0.0000 & 0.8532 & \\
\hline
\end{tabular}

a Standard uncertainty $(u)$ are $u(y)=0.001, u(x)=0.001, u\left(x_{\mathrm{I}}\right)=0.02, u\left(x_{\mathrm{II}}\right)=0.002, u_{\mathrm{r}}(P)=0.02$, and $u(T)=0.1 \mathrm{~K}$ 
Table S3. Continued

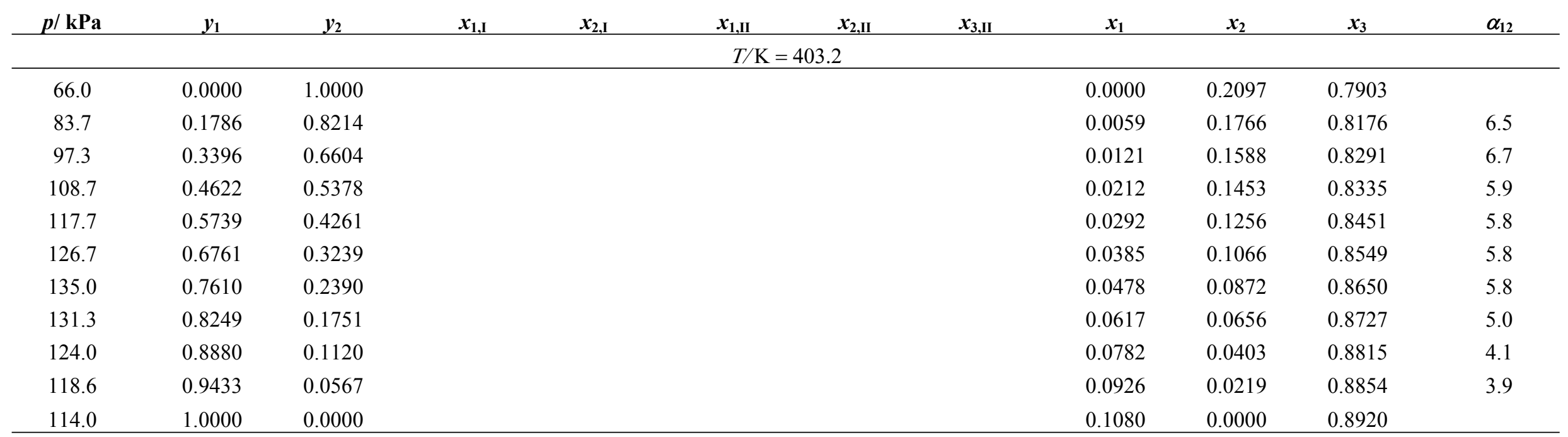

${ }^{a}$ Standard uncertainty $(u)$ are $u(y)=0.001, u(x)=0.001, u\left(x_{\mathrm{I}}\right)=0.02, u\left(x_{\mathrm{II}}\right)=0.002, u_{\mathrm{r}}(P)=0.02$, and $u(T)=0.1 \mathrm{~K}$. 
Table S4. Vapor-liquid and Vapor-liquid-liquid Equilibria ${ }^{a}$ for $n$-Octane (1) + Toluene (2) $+\left[C_{2} C_{1}\right.$ im] $][C M](3)$

\begin{tabular}{|c|c|c|c|c|c|c|c|c|c|c|c|}
\hline$p / \mathbf{k P a}$ & $y_{1}$ & $y_{2}$ & $x_{1, \mathrm{I}}$ & $x_{2, \mathrm{I}}$ & $x_{1, \mathrm{II}}$ & $x_{2, \text { II }}$ & $x_{3, \text { II }}$ & $x_{1}$ & $x_{2}$ & $x_{3}$ & $\alpha_{12}$ \\
\hline \multicolumn{12}{|c|}{$T / \mathrm{K}=323.2$} \\
\hline 4.7 & 0.0000 & 1.0000 & & & & & & 0.0000 & 0.1807 & 0.8193 & \\
\hline 8.5 & 0.5036 & 0.4964 & & & & & & 0.0123 & 0.1669 & 0.8208 & 13.8 \\
\hline 8.8 & 0.5744 & 0.4256 & 0.7295 & 0.2705 & 0.0057 & 0.1426 & 0.8516 & 0.0262 & 0.1463 & 0.8275 & 7.5 \\
\hline 8.5 & 0.6161 & 0.3839 & 0.7689 & 0.2311 & 0.0058 & 0.1228 & 0.8714 & 0.0412 & 0.1279 & 0.8309 & 5.0 \\
\hline 8.3 & 0.6606 & 0.3394 & 0.8046 & 0.1954 & 0.0058 & 0.1040 & 0.8902 & 0.0570 & 0.1099 & 0.8331 & 3.8 \\
\hline 8.0 & 0.7118 & 0.2882 & 0.8379 & 0.1621 & 0.0058 & 0.0860 & 0.9082 & 0.0707 & 0.0920 & 0.8373 & 3.2 \\
\hline 7.8 & 0.7599 & 0.2401 & 0.8699 & 0.1301 & 0.0057 & 0.0679 & 0.9264 & 0.0867 & 0.0738 & 0.8395 & 2.7 \\
\hline 7.6 & 0.8171 & 0.1829 & 0.9018 & 0.0982 & 0.0055 & 0.0500 & 0.9445 & 0.1009 & 0.0551 & 0.8439 & 2.4 \\
\hline 7.3 & 0.8696 & 0.1304 & 0.9339 & 0.0661 & 0.0055 & 0.0335 & 0.9610 & 0.1159 & 0.0374 & 0.8467 & 2.2 \\
\hline 7.1 & 0.9300 & 0.0700 & 0.9673 & 0.0327 & 0.0055 & 0.0166 & 0.9779 & 0.1321 & 0.0187 & 0.8492 & 1.9 \\
\hline 6.7 & 1.0000 & 0.0000 & 1.0000 & 0.0000 & 0.0057 & 0.0000 & 0.9943 & 0.1428 & 0.0000 & 0.8572 & \\
\hline \multicolumn{12}{|c|}{$T / \mathrm{K}=363.2$} \\
\hline 23.8 & 0.0000 & 1.0000 & & & & & & 0.0000 & 0.1730 & 0.8270 & \\
\hline 35.2 & 0.4577 & 0.5423 & & & & & & 0.0080 & 0.1537 & 0.8383 & 16.3 \\
\hline 41.3 & 0.5929 & 0.4071 & 0.7322 & 0.2678 & 0.0058 & 0.1362 & 0.8580 & 0.0197 & 0.1383 & 0.8420 & 10.2 \\
\hline 39.6 & 0.6537 & 0.3463 & 0.7698 & 0.2302 & 0.0060 & 0.1158 & 0.8782 & 0.0354 & 0.1199 & 0.8447 & 6.4 \\
\hline 39.0 & 0.6908 & 0.3092 & 0.8132 & 0.1868 & 0.0061 & 0.0984 & 0.8955 & 0.0529 & 0.1033 & 0.8438 & 4.4 \\
\hline 37.9 & 0.7439 & 0.2561 & 0.8501 & 0.1499 & 0.0062 & 0.0808 & 0.9129 & 0.0667 & 0.0855 & 0.8477 & 3.7 \\
\hline 37.0 & 0.7938 & 0.2062 & 0.8787 & 0.1213 & 0.0063 & 0.0640 & 0.9297 & 0.0844 & 0.0689 & 0.8467 & 3.1 \\
\hline 36.4 & 0.8413 & 0.1587 & 0.9109 & 0.0891 & 0.0059 & 0.0476 & 0.9465 & 0.1003 & 0.0517 & 0.8479 & 2.7 \\
\hline 35.6 & 0.8911 & 0.1089 & 0.9414 & 0.0586 & 0.0063 & 0.0321 & 0.9617 & 0.1164 & 0.0351 & 0.8485 & 2.5 \\
\hline 34.7 & 0.9430 & 0.0570 & 0.9708 & 0.0292 & 0.0065 & 0.0156 & 0.9779 & 0.1325 & 0.0174 & 0.8501 & 2.2 \\
\hline 33.4 & 1.0000 & 0.0000 & 1.0000 & 0.0000 & 0.0066 & 0.0000 & 0.9934 & 0.1468 & 0.0000 & 0.8532 & \\
\hline
\end{tabular}

a Standard uncertainty $(u)$ are $u(y)=0.001, u(x)=0.001, u\left(x_{\mathrm{I}}\right)=0.02, u\left(x_{\mathrm{II}}\right)=0.002, u_{\mathrm{r}}(P)=0.02$, and $u(T)=0.1 \mathrm{~K}$. 
Table S4. Continued

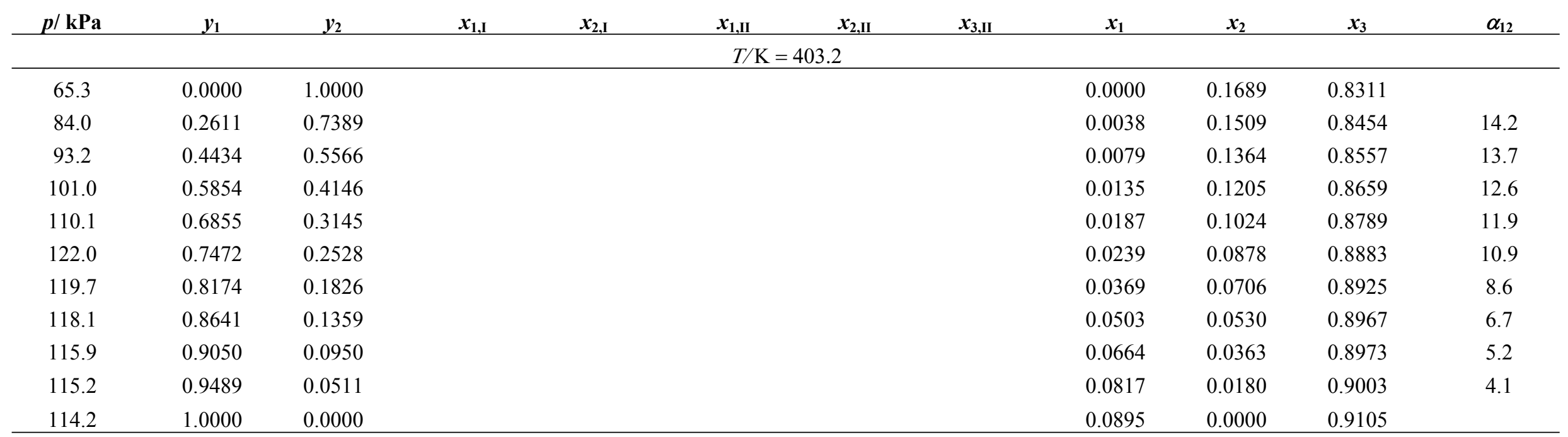

${ }^{a}$ Standard uncertainty $(u)$ are $u(y)=0.001, u(x)=0.001, u\left(x_{\mathrm{I}}\right)=0.02, u\left(x_{\mathrm{II}}\right)=0.002, u_{\mathrm{r}}(P)=0.02$, and $u(T)=0.1 \mathrm{~K}$. 
Table S5. Vapor-liquid and Vapor-liquid-liquid Equilibria ${ }^{a}$ for $n$-Octane (1) + Toluene (2) $+\left[4-C_{4} C_{1}\right.$ py $][$ TCM] (3)

\begin{tabular}{|c|c|c|c|c|c|c|c|c|c|c|c|}
\hline$p / \mathbf{k P a}$ & $y_{1}$ & $y_{2}$ & $x_{1, \mathrm{I}}$ & $x_{2, \mathrm{I}}$ & $x_{1, \mathrm{II}}$ & $x_{2, \mathrm{II}}$ & $x_{3, \mathrm{II}}$ & $x_{1}$ & $x_{2}$ & $x_{3}$ & $\alpha_{12}$ \\
\hline \multicolumn{12}{|c|}{$T / \mathrm{K}=323.2$} \\
\hline 3.4 & 0.0000 & 1.0000 & & & & & & 0.0000 & 0.2092 & 0.7908 & \\
\hline 6.7 & 0.5668 & 0.4332 & & & & & & 0.0150 & 0.1971 & 0.7878 & 17.1 \\
\hline 8.2 & 0.6874 & 0.3126 & 0.7863 & 0.2137 & 0.0103 & 0.1739 & 0.8158 & 0.0308 & 0.1750 & 0.7943 & 12.5 \\
\hline 8.0 & 0.7281 & 0.2719 & 0.8189 & 0.1811 & 0.0103 & 0.1493 & 0.8404 & 0.0478 & 0.1507 & 0.8014 & 8.4 \\
\hline 7.9 & 0.7563 & 0.2437 & 0.8414 & 0.1586 & 0.0101 & 0.1284 & 0.8614 & 0.0670 & 0.1305 & 0.8025 & 6.0 \\
\hline 7.6 & 0.7940 & 0.2060 & 0.8668 & 0.1332 & 0.0102 & 0.1087 & 0.8811 & 0.0845 & 0.1108 & 0.8047 & 5.1 \\
\hline 7.5 & 0.8281 & 0.1719 & 0.8948 & 0.1052 & 0.0102 & 0.0858 & 0.9041 & 0.1025 & 0.0878 & 0.8097 & 4.1 \\
\hline 7.2 & 0.8697 & 0.1303 & 0.9219 & 0.0781 & 0.0113 & 0.0647 & 0.9240 & 0.1208 & 0.0663 & 0.8129 & 3.7 \\
\hline 7.1 & 0.9085 & 0.0915 & 0.9464 & 0.0536 & 0.0104 & 0.0439 & 0.9457 & 0.1395 & 0.0452 & 0.8153 & 3.2 \\
\hline 7.0 & 0.9509 & 0.0491 & 0.9735 & 0.0265 & 0.0102 & 0.0217 & 0.9681 & 0.1572 & 0.0224 & 0.8203 & 2.8 \\
\hline 6.7 & 1.0000 & 0.0000 & 1.0000 & 0.0000 & 0.0106 & 0.0000 & 0.9894 & 0.1659 & 0.0000 & 0.8341 & \\
\hline \multicolumn{12}{|c|}{$T / \mathrm{K}=363.2$} \\
\hline 14.5 & 0.0000 & 1.0000 & & & & & & 0.0000 & 0.2037 & 0.7963 & \\
\hline 23.9 & 0.4269 & 0.5731 & & & & & & 0.0100 & 0.1837 & 0.8063 & 13.6 \\
\hline 33.1 & 0.6180 & 0.3820 & & & & & & 0.0199 & 0.1661 & 0.8139 & 13.5 \\
\hline 38.4 & 0.7323 & 0.2677 & 0.8248 & 0.0270 & 0.0099 & 0.1367 & 0.8534 & 0.0293 & 0.1380 & 0.8327 & 12.9 \\
\hline 37.7 & 0.7627 & 0.2373 & 0.8454 & 0.0270 & 0.0103 & 0.1225 & 0.8672 & 0.0500 & 0.1244 & 0.8256 & 8.0 \\
\hline 37.4 & 0.7932 & 0.2068 & 0.8695 & 0.0270 & 0.0103 & 0.1028 & 0.8870 & 0.0662 & 0.1050 & 0.8288 & 6.1 \\
\hline 36.2 & 0.8354 & 0.1646 & 0.8959 & 0.0270 & 0.0108 & 0.0816 & 0.9077 & 0.0841 & 0.0837 & 0.8322 & 5.1 \\
\hline 35.7 & 0.8709 & 0.1291 & 0.9207 & 0.0270 & 0.0113 & 0.0617 & 0.9270 & 0.1027 & 0.0637 & 0.8336 & 4.2 \\
\hline 35.2 & 0.9121 & 0.0879 & 0.9488 & 0.0270 & 0.0115 & 0.0414 & 0.9471 & 0.1181 & 0.0427 & 0.8392 & 3.8 \\
\hline 34.5 & 0.9528 & 0.0472 & 0.9744 & 0.0114 & 0.0116 & 0.0208 & 0.9677 & 0.1377 & 0.0215 & 0.8408 & 3.1 \\
\hline 33.4 & 1.0000 & 0.0000 & 1.0000 & 0.0005 & 0.0116 & 0.0000 & 0.9884 & 0.1502 & 0.0000 & 0.8498 & \\
\hline
\end{tabular}

a Standard uncertainty $(u)$ are $u(y)=0.001, u(x)=0.001, u\left(x_{\mathrm{I}}\right)=0.02, u\left(x_{\mathrm{II}}\right)=0.002, u_{\mathrm{r}}(P)=0.02$, and $u(T)=0.1 \mathrm{~K}$. 
Table S5. Continued

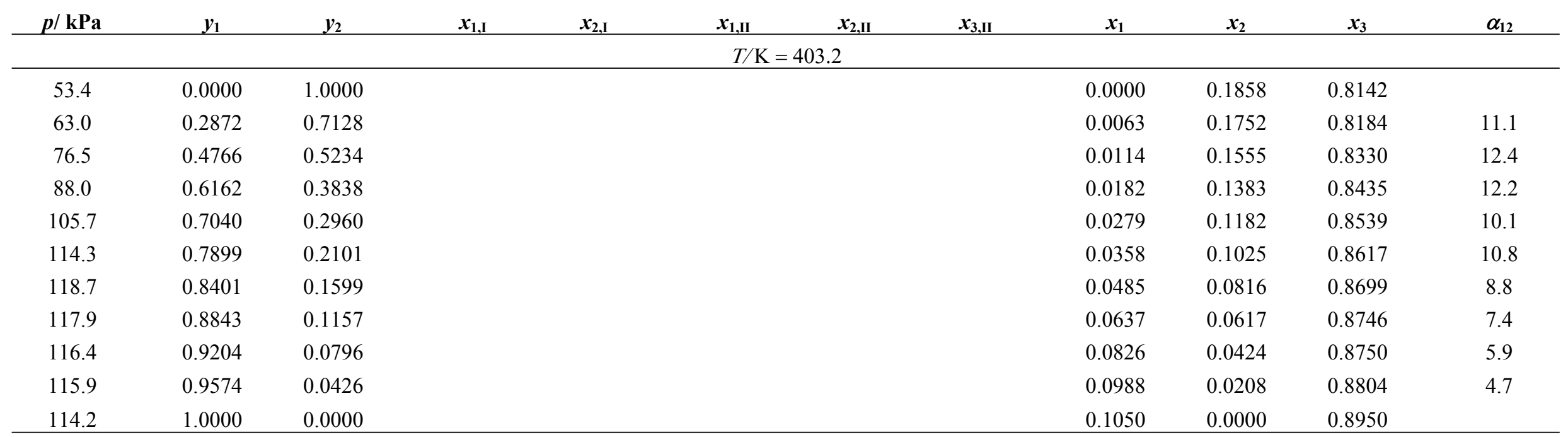

${ }^{a}$ Standard uncertainty $(u)$ are $u(y)=0.001, u(x)=0.001, u\left(x_{\mathrm{I}}\right)=0.02, u\left(x_{\mathrm{II}}\right)=0.002, u_{\mathrm{r}}(P)=0.02$, and $u(T)=0.1 \mathrm{~K}$. 
Table S6. Vapor-liquid and Vapor-liquid-liquid Equilibria ${ }^{a}$ for $n$-Heptane (1) + Benzene (2) $+\left[C_{2} C_{1}\right.$ im $][$ TCM] (3)

\begin{tabular}{|c|c|c|c|c|c|c|c|c|c|c|c|}
\hline$p / \mathbf{k P a}$ & $y_{1}$ & $y_{2}$ & $x_{1, \mathrm{I}}$ & $x_{2, \mathrm{I}}$ & $x_{1, \mathrm{II}}$ & $x_{2, \mathrm{II}}$ & $x_{3, \mathrm{II}}$ & $x_{1}$ & $x_{2}$ & $x_{3}$ & $\alpha_{12}$ \\
\hline \multicolumn{12}{|c|}{$T / \mathrm{K}=323.2$} \\
\hline 8.9 & 0.0000 & 1.0000 & & & & & & 0.0000 & 0.1963 & 0.8037 & \\
\hline 20.2 & 0.5356 & 0.4644 & & & & & & 0.0096 & 0.1814 & 0.8090 & 21.8 \\
\hline 23.0 & 0.6366 & 0.3634 & 0.7943 & 0.2057 & 0.0103 & 0.1604 & 0.8293 & 0.0232 & 0.1620 & 0.8148 & 12.2 \\
\hline 22.5 & 0.6677 & 0.3323 & 0.8157 & 0.1843 & 0.0110 & 0.1428 & 0.8462 & 0.0387 & 0.1449 & 0.8164 & 7.5 \\
\hline 21.9 & 0.7153 & 0.2847 & 0.8452 & 0.1548 & 0.0111 & 0.1235 & 0.8654 & 0.0530 & 0.1257 & 0.8213 & 6.0 \\
\hline 21.6 & 0.7520 & 0.2480 & 0.8739 & 0.1261 & 0.0113 & 0.1006 & 0.8881 & 0.0725 & 0.1030 & 0.8245 & 4.3 \\
\hline 20.9 & 0.7942 & 0.2058 & 0.9015 & 0.0985 & 0.0115 & 0.0789 & 0.9097 & 0.0889 & 0.0811 & 0.8300 & 3.5 \\
\hline 20.4 & 0.8399 & 0.1601 & 0.9265 & 0.0735 & 0.0120 & 0.0594 & 0.9286 & 0.1051 & 0.0612 & 0.8337 & 3.1 \\
\hline 20.0 & 0.8880 & 0.1120 & 0.9505 & 0.0495 & 0.0122 & 0.0395 & 0.9483 & 0.1220 & 0.0409 & 0.8370 & 2.7 \\
\hline 19.6 & 0.9437 & 0.0563 & 0.9736 & 0.0264 & 0.0126 & 0.0213 & 0.9662 & 0.1376 & 0.0221 & 0.8403 & 2.7 \\
\hline 18.9 & 1.0000 & 0.0000 & 1.0000 & 0.0000 & 0.0130 & 0.0000 & 0.9870 & 0.1545 & 0.0000 & 0.8455 & \\
\hline \multicolumn{12}{|c|}{$T / \mathrm{K}=363.2$} \\
\hline 47.2 & 0.0000 & 1.0000 & & & & & & 0.0000 & 0.1740 & 0.8260 & \\
\hline 55.0 & 0.3732 & 0.6268 & & & & & & 0.0039 & 0.1644 & 0.8317 & 24.8 \\
\hline 71.9 & 0.5289 & 0.4711 & & & & & & 0.0099 & 0.1495 & 0.8407 & 17.0 \\
\hline 86.0 & 0.6558 & 0.3442 & & & & & & 0.0136 & 0.1319 & 0.8546 & 18.5 \\
\hline 89.4 & 0.7293 & 0.2707 & 0.8686 & 0.1314 & 0.0103 & 0.1130 & 0.8767 & 0.0224 & 0.1146 & 0.8630 & 13.8 \\
\hline 86.7 & 0.7719 & 0.2281 & 0.8901 & 0.1099 & 0.0102 & 0.0909 & 0.8989 & 0.0399 & 0.0927 & 0.8674 & 7.9 \\
\hline 85.4 & 0.8208 & 0.1792 & 0.9088 & 0.0912 & 0.0101 & 0.0730 & 0.9169 & 0.0564 & 0.0749 & 0.8688 & 6.1 \\
\hline 82.2 & 0.8630 & 0.1370 & 0.9323 & 0.0677 & 0.0107 & 0.0552 & 0.9341 & 0.0723 & 0.0568 & 0.8709 & 4.9 \\
\hline 81.3 & 0.9092 & 0.0908 & 0.9534 & 0.0466 & 0.0106 & 0.0362 & 0.9532 & 0.0859 & 0.0375 & 0.8765 & 4.4 \\
\hline 77.9 & 0.9494 & 0.0506 & 0.9744 & 0.0256 & 0.0110 & 0.0198 & 0.9692 & 0.1051 & 0.0207 & 0.8742 & 3.7 \\
\hline 76.9 & 1.0000 & 0.0000 & 1.0000 & 0.0000 & 0.0125 & 0.0000 & 0.9875 & 0.1236 & 0.0000 & 0.8764 & \\
\hline
\end{tabular}

a Standard uncertainty $(u)$ are $u(y)=0.001, u(x)=0.001, u\left(x_{\mathrm{I}}\right)=0.02, u\left(x_{\mathrm{II}}\right)=0.002, u_{\mathrm{r}}(P)=0.02$, and $u(T)=0.1 \mathrm{~K}$. 
Table S6. Continued

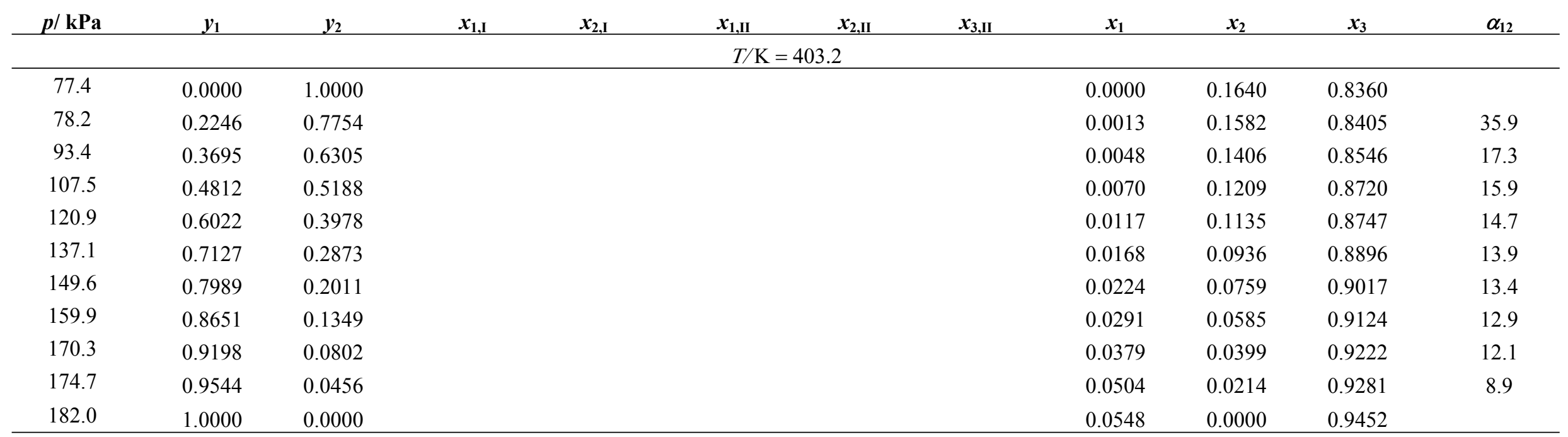

${ }^{a}$ Standard uncertainty $(u)$ are $u(y)=0.001, u(x)=0.001, u\left(x_{\mathrm{I}}\right)=0.02, u\left(x_{\mathrm{II}}\right)=0.002, u_{\mathrm{r}}(P)=0.02$, and $u(T)=0.1 \mathrm{~K}$. 
Table S7. Vapor-liquid and Vapor-liquid-liquid Equilibria ${ }^{a}$ for $n$-Heptane (1) + Benzene (2) $+\left[4-C_{4} C_{1}\right.$ py $][$ TCM] (3)

\begin{tabular}{|c|c|c|c|c|c|c|c|c|c|c|c|}
\hline$p / \mathbf{k P a}$ & $y_{1}$ & $y_{2}$ & $x_{1, \mathrm{I}}$ & $x_{2, \mathrm{I}}$ & $x_{1, \mathrm{II}}$ & $x_{2, \mathrm{II}}$ & $x_{3, \mathrm{II}}$ & $x_{1}$ & $x_{2}$ & $x_{3}$ & $\alpha_{12}$ \\
\hline \multicolumn{12}{|c|}{$T / \mathrm{K}=323.2$} \\
\hline 9.1 & 0.0000 & 1.0000 & & & & & & 0.0000 & 0.2319 & 0.7681 & \\
\hline 13.1 & 0.4908 & 0.5092 & & & & & & 0.0145 & 0.2131 & 0.7724 & 14.1 \\
\hline 20.0 & 0.6928 & 0.3072 & & & & & & 0.0273 & 0.1889 & 0.7838 & 15.6 \\
\hline 22.0 & 0.7567 & 0.2433 & 0.8423 & 0.1577 & 0.0227 & 0.1672 & 0.8101 & 0.0428 & 0.1677 & 0.7895 & 12.2 \\
\hline 21.6 & 0.7855 & 0.2145 & 0.8649 & 0.1351 & 0.0239 & 0.1462 & 0.8299 & 0.0600 & 0.1463 & 0.7936 & 8.9 \\
\hline 21.0 & 0.8182 & 0.1818 & 0.8925 & 0.1075 & 0.0246 & 0.1190 & 0.8564 & 0.0817 & 0.1187 & 0.7995 & 6.5 \\
\hline 20.6 & 0.8485 & 0.1515 & 0.9135 & 0.0865 & 0.0244 & 0.0948 & 0.8808 & 0.1018 & 0.0945 & 0.8037 & 5.2 \\
\hline 20.0 & 0.8835 & 0.1165 & 0.9344 & 0.0656 & 0.0250 & 0.0713 & 0.9037 & 0.1200 & 0.0710 & 0.8090 & 4.5 \\
\hline 19.9 & 0.9166 & 0.0834 & 0.9537 & 0.0463 & 0.0254 & 0.0479 & 0.9267 & 0.1412 & 0.0480 & 0.8108 & 3.7 \\
\hline 19.5 & 0.9579 & 0.0421 & 0.9744 & 0.0256 & 0.0263 & 0.0260 & 0.9477 & 0.1610 & 0.0261 & 0.8129 & 3.7 \\
\hline 19.2 & 1.0000 & 0.0000 & 1.0000 & 0.0000 & 0.0261 & 0.0000 & 0.9739 & 0.1818 & 0.0000 & 0.8182 & \\
\hline \multicolumn{12}{|c|}{$T / \mathrm{K}=363.2$} \\
\hline 25.8 & 0.0000 & 1.0000 & & & & & & 0.0000 & 0.2075 & 0.7925 & \\
\hline 38.7 & 0.3316 & 0.6684 & & & & & & 0.0092 & 0.1889 & 0.8018 & 10.2 \\
\hline 46.1 & 0.5051 & 0.4949 & & & & & & 0.0219 & 0.2371 & 0.7411 & 11.1 \\
\hline 61.4 & 0.6627 & 0.3373 & & & & & & 0.0233 & 0.1499 & 0.8268 & 12.6 \\
\hline 73.2 & 0.7649 & 0.2351 & & & & & & 0.0279 & 0.1304 & 0.8417 & 15.2 \\
\hline 83.9 & 0.8304 & 0.1696 & 0.8974 & 0.1026 & 0.0216 & 0.1097 & 0.8687 & 0.0423 & 0.1106 & 0.8470 & 12.8 \\
\hline 83.0 & 0.8711 & 0.1289 & 0.9179 & 0.0821 & 0.0217 & 0.0858 & 0.8925 & 0.0579 & 0.0865 & 0.8555 & 10.1 \\
\hline 81.4 & 0.8991 & 0.1009 & 0.9359 & 0.0641 & 0.0217 & 0.0657 & 0.9126 & 0.0776 & 0.0663 & 0.8562 & 7.6 \\
\hline 81.5 & 0.9324 & 0.0676 & 0.9575 & 0.0425 & 0.0220 & 0.0439 & 0.9341 & 0.0948 & 0.0443 & 0.8609 & 6.4 \\
\hline 78.5 & 0.9630 & 0.0370 & 0.9800 & 0.0200 & 0.0220 & 0.0196 & 0.9584 & 0.1129 & 0.0238 & 0.8633 & 5.5 \\
\hline 76.4 & 1.0000 & 0.0000 & 1.0000 & 0.0000 & 0.0237 & 0.0000 & 0.9763 & 0.1353 & 0.0000 & 0.8647 & \\
\hline
\end{tabular}

a Standard uncertainty $(u)$ are $u(y)=0.001, u(x)=0.001, u\left(x_{\mathrm{I}}\right)=0.02, u\left(x_{\mathrm{II}}\right)=0.002, u_{\mathrm{r}}(P)=0.02$, and $u(T)=0.1 \mathrm{~K}$. 
Table S7. Continued

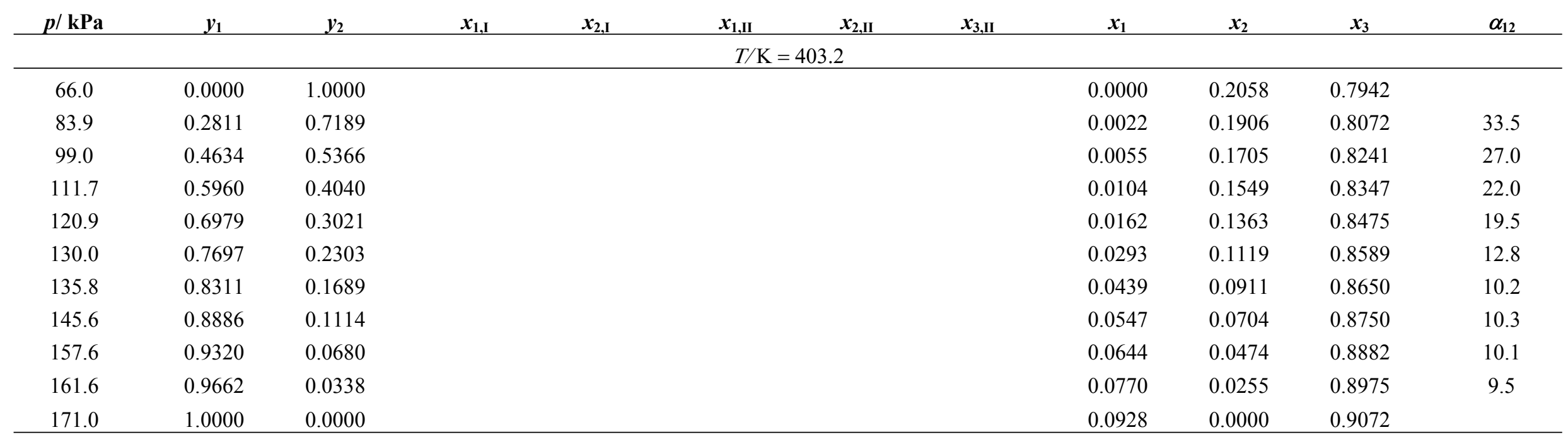

${ }^{a}$ Standard uncertainty $(u)$ are $u(y)=0.001, u(x)=0.001, u\left(x_{\mathrm{I}}\right)=0.02, u\left(x_{\mathrm{II}}\right)=0.002, u_{\mathrm{r}}(P)=0.02$, and $u(T)=0.1 \mathrm{~K}$. 

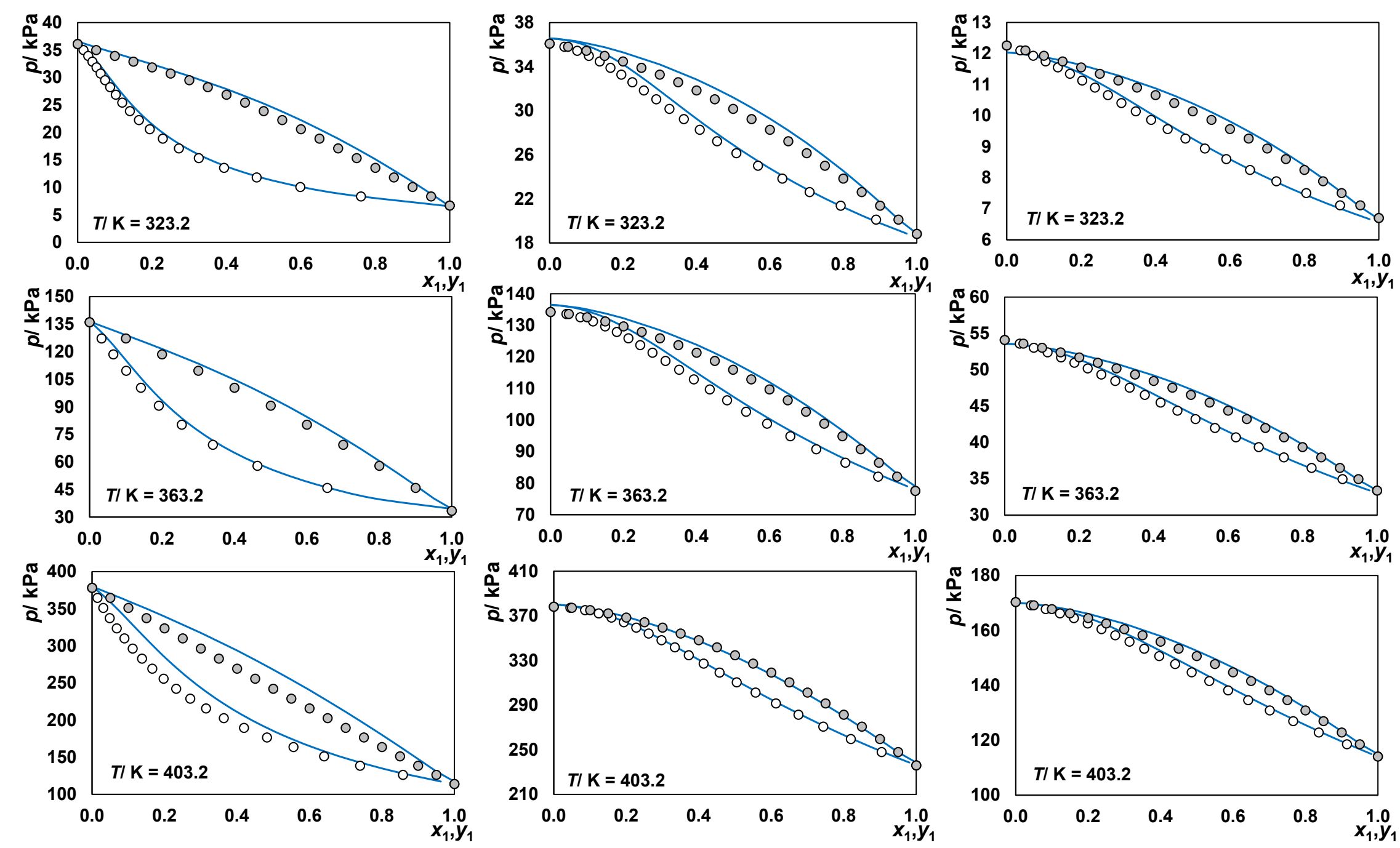

Figure S1. VLE Diagrams (p-x,y) for the Binary Mixtures: $n$-Octane (1) + Benzene (2), Left; $n$-Heptane (1) + Benzene (2), Middle; $n$-Octane (1) + Toluene (2), Right. Experimental Data from Ref ${ }^{48}$ and CPA EoS Description Using Binary Interaction Parameters from Table S1. 

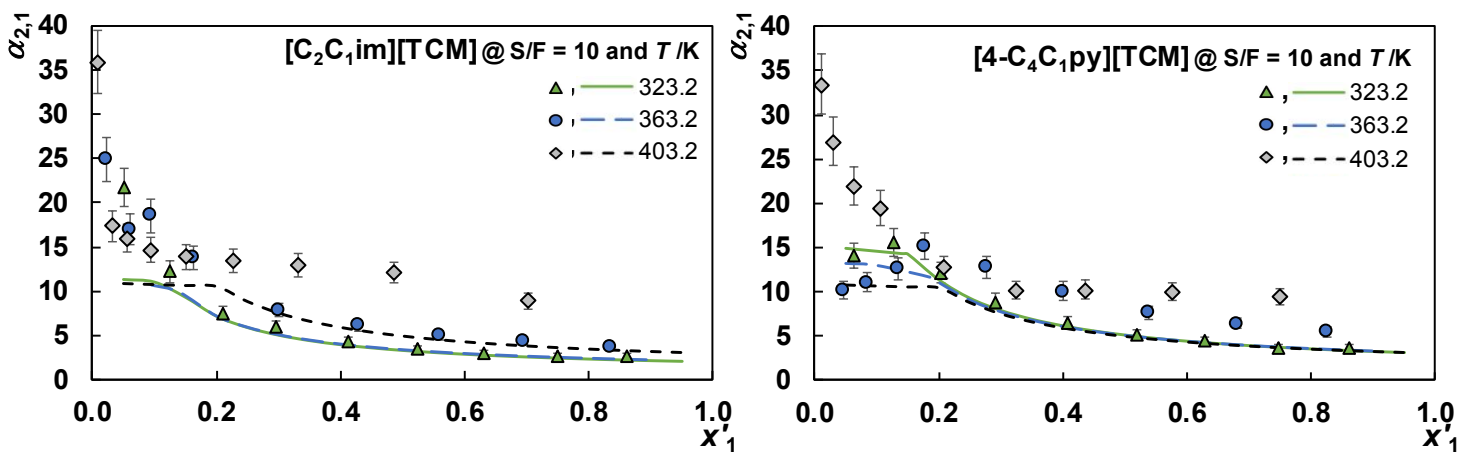

Figure S2. $n$-Heptane/benzene Relative Volatility as Function of the Free-IL Aliphatic Mole Fraction for the $\left\{n\right.$-Octane + Benzene $+\left[\mathrm{C}_{2} \mathrm{C}_{1} \mathrm{im}\right][\mathrm{TCM}] /\left[4-\mathrm{C}_{4} \mathrm{C}_{1}\right.$ py $\left.][\mathrm{TCM}]\right\}$. Symbols Represent the Experimental Data and the Lines the CPA EoS. 

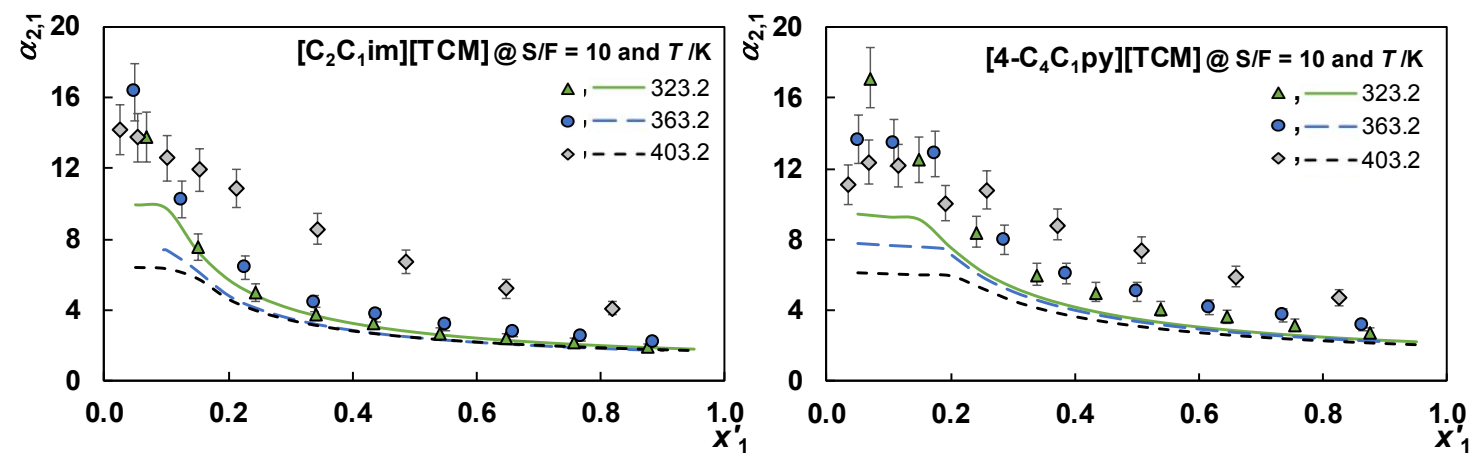

Figure S3. $n$-Octane/toluene Relative Volatility as Function of the Free-IL Aliphatic Mole Fraction for the $\left\{n\right.$-Octane + Benzene $+\left[\mathrm{C}_{2} \mathrm{C}_{1}\right.$ im $][\mathrm{TCM}] /\left[4-\mathrm{C}_{4} \mathrm{C}_{1}\right.$ py $\left.][\mathrm{TCM}]\right\}$. Symbols Represent the Experimental Data and the Lines the CPA EoS. 